\title{
Agent Based Modeling and Simulation of Tsunami Triggered Mass Evacuation Considering Changes of Environment Due to Earthquake and Inundation
}

\author{
Stephen JACOB ${ }^{1}$, Leonel AGUILAR ${ }^{2}$, Lalith WIJERATHNE ${ }^{3}$, Muneo HORI ${ }^{4}$, \\ Tsuyoshi ICHIMURA $^{5}$, and Seizo TANAKA ${ }^{6}$ \\ ${ }^{1}$ Civil \& Building Department, JGC Corporation, Minato mirai, Yokohama \\ E-mail: philips_jacob.stephen@jgc.com \\ ${ }^{2} \mathrm{PhD}$. Candidate, Department of Civil Engineering, University of Tokyo (Bunkyo, Tokyo 113-0032) \\ E-mail: aguilar@eri.u-tokyo.ac.jp \\ ${ }^{3}$ Member of JSCE, Assoc. Prof. Earthquake Research Institute, University of Tokyo (Bunkyo, Tokyo 113-0032) \\ E-mail: lalith@eri.u-tokyo.ac.jp \\ ${ }^{4}$ Member of JSCE, Prof. Earthquake Research Institute, University of Tokyo (Bunkyo, Tokyo 113-0032) \\ E-mail: hori@eri.u-tokyo.ac.jp \\ ${ }^{5}$ Member of JSCE, Assoc. Prof. Earthquake Research Institute, University of Tokyo (Bunkyo, Tokyo 113-0032) \\ E-mail: ichimura@eri.u-tokyo.ac.jp \\ ${ }^{6}$ Member of JSCE, Asst. Prof. Earthquake Research Institute, University of Tokyo (Bunkyo, Tokyo 113-0032) \\ E-mail: stanaka@eri.u-tokyo.ac.jp
}

\begin{abstract}
An agent based model, which includes detailed model of the environment and agents with functionalities to interact with it, is developed for simulating large area evacuations. This model makes it possible to quantitatively estimate the effects of various important factors, like interaction with environment and neighbor agents in low lighting conditions, vehicle-pedestrian interaction in narrow roads, etc., which cannot be modeled with the existing models. The evacuation simulator is coupled with an integrated earthquake simulator to take the earthquake damages into account. To demonstrate the potential of the model, several scenarios, which require detailed modeling of the environment, are presented.
\end{abstract}

Key Words: tsunami, evacuation, earthquake damages, low visibility, agent based modeling and simulation

\section{Introduction}

Quantitative evaluation of various factors, which may significantly affect evacuation time, is of great importance in time critical mass evacuations like those triggered by tsunamis. Agent based modeling is one of the widely used numerical method in studying mass evacuations; out of methods like cellular automata, social force model, etc., agent based models are preferred since it can model complex actions of evacuees in heterogeneous crowds. The existing agent based large area evacuation simulators use simplified models of environment and agents; e.g., environment is modeled as a topological network of available roads. Though these simulators provide many useful information, they have inherent limitations. Some of the important factors requiring a detailed model of the environment are the effects of earthquake induced damages, visitors following others, interactions with environment and neighbor agents in low lighting conditions, localized dam- ages of the environment, vehicle-pedestrian interaction in narrow roads, etc. According to the authors' knowledge, there are no records of research which consider the above mentioned factors requiring a detailed model of the environment. It is of great interest to quantitatively estimate the effects of these factors on tsunami triggered evacuations, especially when the tsunami arrival time is short.

With the aim of exploring the effectiveness of different strategies to make the evacuation process, in large urban areas, smooth and fast, we are developing an agent based evacuation simulation code ${ }^{1)}$. In order to overcome the limitations of existing mass evacuation simulators, a detailed model of the environment and agents with functionalities to interact with it and other agents are included. The environment is modeled as a hybrid of a high resolution grid and a topological graph. The evacuation simulator is coupled with the Integrated Earthquake Simulator (IES) ${ }^{2}$ and tsunami simulation software in order to automatically include earthquake induced building damages. Fur- 
ther, the grid is dynamically updated according to the results of the tsunami inundation simulation, this way considering the effects of earthquake and tsunami disasters. As a demonstrative application, requiring detailed model of environment, the effect of lighting on night time evacuation during a hypothetical festival in a coastal city and some other scenario simulations are presented. A brief description of our agent based model is presented in the second section. The third section provides demonstrative applications of the presented simulator in tsunami triggered emergency evacuation of large urban areas. This demonstrative applications are presented in order to highlight the need of detailed models and the ability to quantitatively estimate their effect.

\section{Agent Based Model}

Out of several numerical techniques for evacuation simulations, agent based simulation is selected since it is capable of simulating complex states emerging due to the heterogeneity in crowds. In agent based simulation, agents represent the evacuees. The usefulness of this modeling technique stems from the ability of assigning heterogeneous properties to each agent and each of them being programmed to take relevant actions according to their specific circumstances and the roles they play, e.g., the action of police officers and volunteers in supporting groups of visitors to reach a safe place. The agent based model consists of two major components; agents and the environment. The major differences of the agent based model presented in this paper, compared to existing models, are the use of high resolution environment and agents capable of perceiving and interacting with it. In this section, the details of the environment model and its construction, as well as some details of the interaction of the agents with it, are presented.

\section{(1) Model of the environment}

The environment is modeled as a hybrid of a high resolution two dimensional grid and a topological graph with its edges representing available paths (see Fig. 1). This hybrid raster/vector model is chosen to conveniently exploit the strengths of each paradigm. Generation of both models is automated since manual generation is tedious and error prone for the domains of several tens to hundreds of square kilometers.

The main purpose of the high resolution $2 \mathrm{D}$ grid is to overcome the limitations of the existing large area evacuation simulators, by including a detailed model of the environment; and agents able to perceive and interact with it. The grid enables the inclusion of localized dynamic changes like tsunami inundation and earthquake damages, and modeling of localized be-

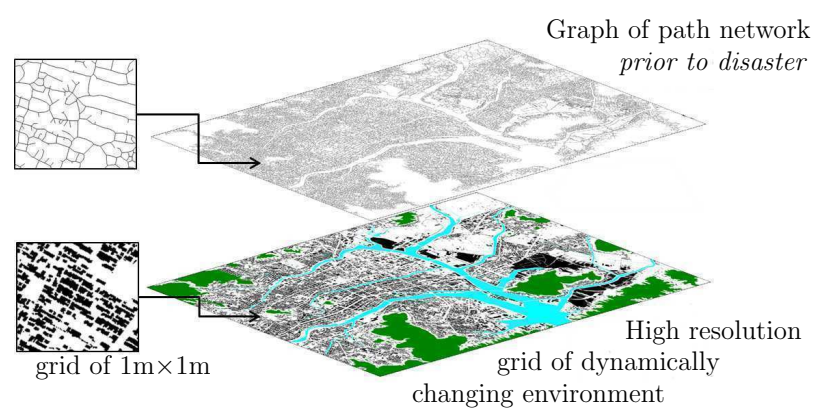

Fig.1 Hybrid model of grid and graph environment. Grid is dynamically updated, reflecting changes in the environment. The graph is static and represents the path network before a disaster.

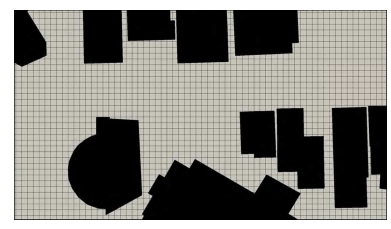

(a) input grid

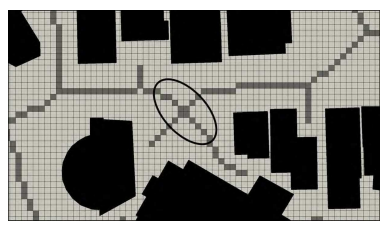

(c) single pixel skeleton

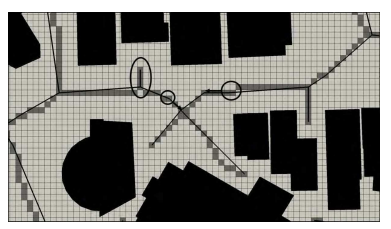

(e) pruning and merging

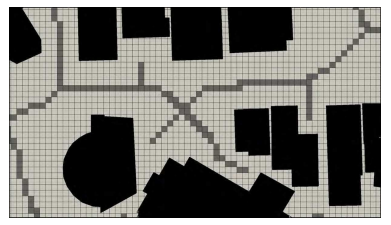

(b) output from EVG-thin

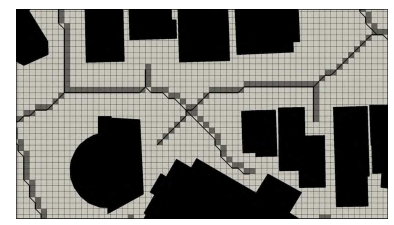

(d) crude vector graph

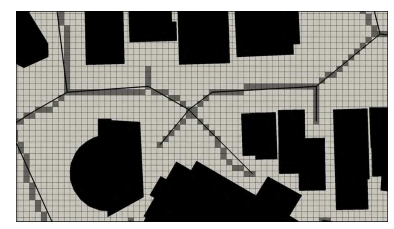

(f) final vector graph
Fig.2 An example demonstrating the steps involved in automated generation of graph.

haviors like crowd forming, traffic pedestrian interactions, etc. The grid is automatically generated from GIS data using a flood fill algorithm. Currently the default cell size resolution is set to $1 \times 1 \mathrm{~m}^{2}$. But the grid resolution can be selected based on the target application and desired detail. The cells hold one of three possible states: occupied, unoccupied or exit.

The topological graph is useful from the performance stand point to allow the agents to perform path planning with lower computational cost. Additionally from the modeling point of view the graph plays a central role in simulating evacuation in earthquake damaged and/or tsunami inundated environment. The topological graph represents the mental map of agent and is used to collect their experiences and takes those into account in path planning. This enables the implementation of strategic path planning for official 
agents, or path re-planing when the agents realize that their desired roads are blocked. Finally from the data gathering point of view it is useful for collecting simulation statistics and post processing.

The graph is automatically constructed from the grid environment so that the graph and the grid are compatible. The automated construction of the graphs involves the following steps.

Thinning: Obtain a pixel skeleton connecting unoccupied spaces by pixel thinning (Fig. 2b); used EVG-Thin ${ }^{4}$ by Beeson.

Reduction of pixel skeleton: Reduce the above pixel skeleton to 1 pixel width (Fig. 2c).

Generation of a crude graph: Add nodes at every branching point of the pixel skeleton, at locations where the width changes rapidly, and at every $25 \mathrm{~m}$ intervals (Fig. 2d).

Prune and merge: Prune childless branches shorter than $5 \mathrm{~m}$, and merge the nodes within $5 \mathrm{~m}$ neighborhood (Fig. 2e).

Assign states of nodes: State of a node is set to exit, if there are grid cells with state of exit, within $50 \mathrm{~m}$ visible distance.

The second step is included since extracting vector graph generation in step 3 involves less processing with a single pixel skeleton. The process of reducing to a single pixel skeleton is automated using a set of templates for identifying and correcting parts wider than a single pixel. In the third step, nodes at $25 \mathrm{~m}$ interval, half of the average sight distance of an agent, are introduced as required for the agents' navigation in damaged environment. Nodes of the graph can have two states, exit or not, while the links have several information like the width, number of each type of agents close to each link, number of agents already passed, etc.

\section{a) Modeling of tsunami inundation and earth- quake damaged environment}

As mentioned, the objective of this research is to overcome the limitations of the existing simplified models by including a detailed model of the environment. To model the earthquake damages due to a given ground motion, the Seismic Response Analysis tool (SRA), part of the Integrated Earthquake Simulator (IES) ${ }^{2}$, which is a physics based structural seismic response simulator capable of simulating a large urban area, is utilized. The flowchart in Fig. 3 shows the process of the physics based modeling of the damaged environment. If the structural response exceeds a particular critical threshold inter-story drift value, the structures are deemed damaged and the space occupied by these structure is increased uniformly by $40 \%{ }^{6}$ ), see Fig. 4. In order to model the tsunami inundation, the grid is dynamically updated at a given time interval, according to provided inundation data.

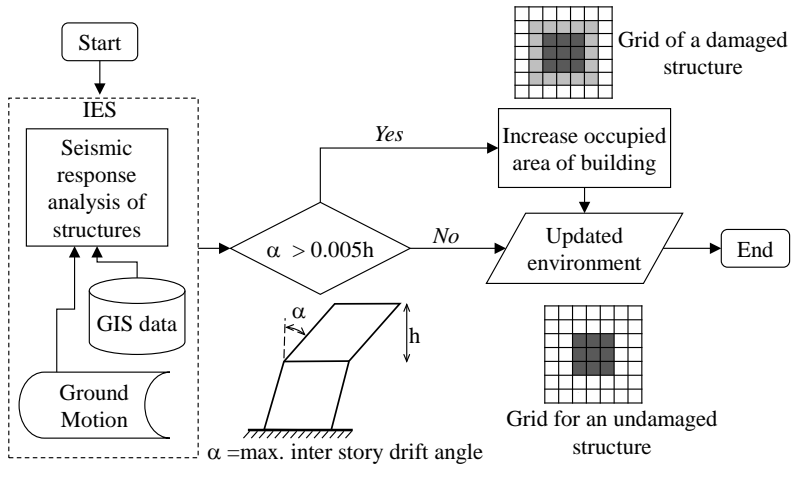

Fig.3 Generation of damaged grid environment.

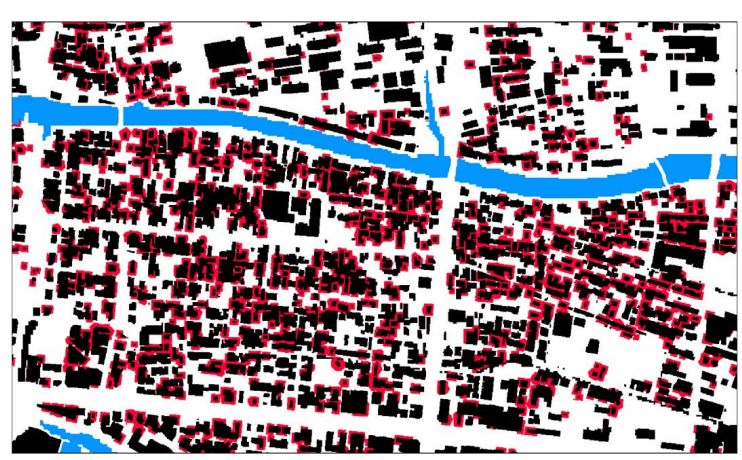

Fig.4 Damaged environment generated according to physics based seismic response analysis; shown in black and red are space occupied by undamaged buildings and debris.

\section{(2) Agents}

Agents represent people evacuating on foot or by vehicles, and interact with each other and the environment to attain their goal of reaching or helping others to reach a safe area. Presently, four basic types of agents are included; resident, visitor, official and car. A simplified flowchart representing agent's execution is presented in Fig. 5. In order to make the agents use the high resolution grid models of complex urban environments, vision functionality is implemented ${ }^{1}$. Agents vision scans the environment like a radar, in high resolution, and deduces the available ways out in their visible surrounding, see Fig. 6. All the agent types, except the visitor, use the topological graph to find paths to a safe destination. The visitor agents do not have access to the graph, and either follow other moving agents in their vicinity or autonomously navigate towards far visible destinations deducing the path based on what they see in their visible surrounding ${ }^{1)}$.

For the purpose of visitor agent's following, it is assumed that, when choosing a person to be followed, the visitors do not know if the person they are following know their destination, i.e. identify residents or people who know the way to an evacuation area. Under this assumption, visitor agents choose to follow 


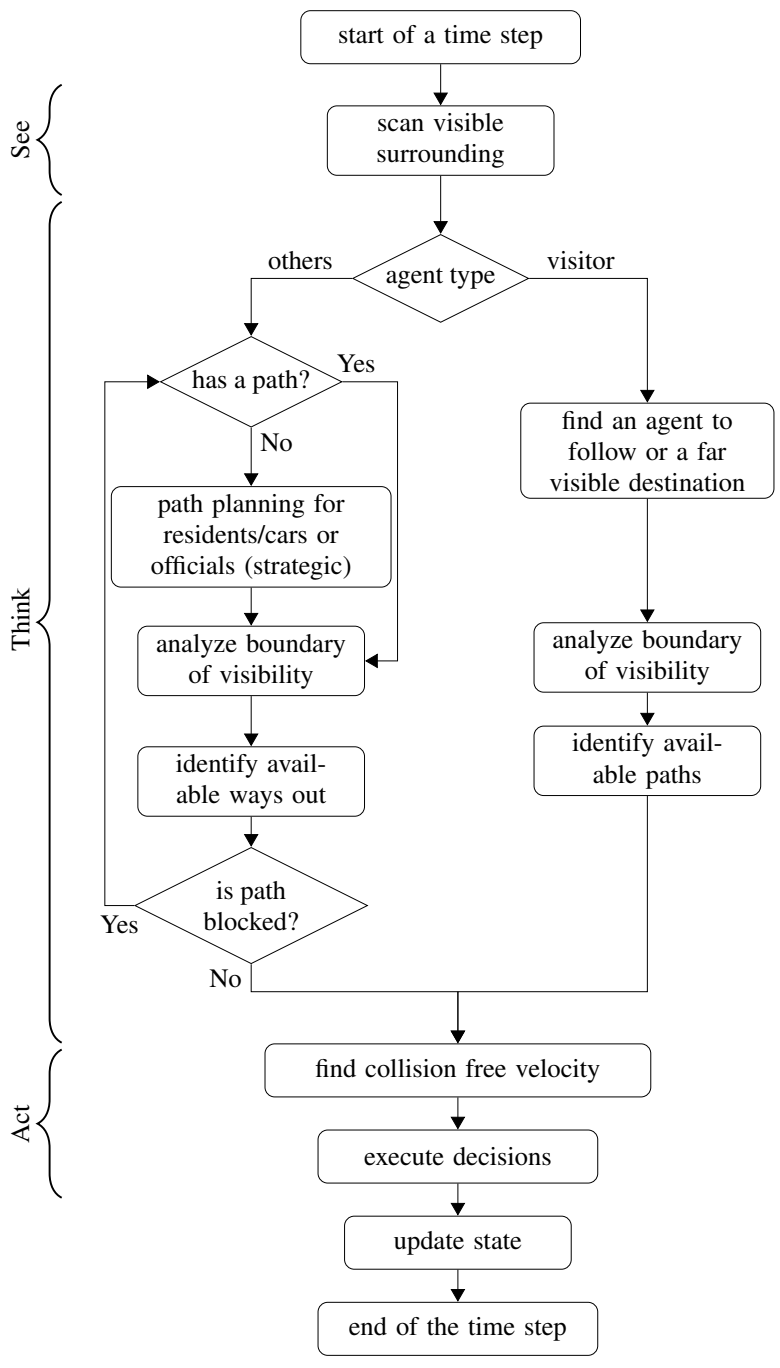

Fig.5 A simplified flowchart of an agents execution.

any moving neighbor agent, which has closest speed to its own. When following, visitor agent first finds the intersecting point of its boundary of visibility and the extension of followee's moving direction, and set this as a temporary target towards which they move. Temporary targets, instead of the followee's location, are used to prevent possible overcrowding when several visitors choose to follow a single agent.

\section{a) Interaction of the agent with the earthquake damaged environment}

Right after an earthquake, none of the evacuees are aware of the damages to the environment, except those they encountered while evacuating. In order to mimic this fact, only the grid is updated according to the damages estimated from IES and/or inundation data. Analyzing the data from their vision functionality, the residents identify any blocked paths. When a blocked path is encountered, the resident agents replan their paths using the topological graph of the undamaged environment and any relevant past experiences like blocked paths stored in their memory. Nav-

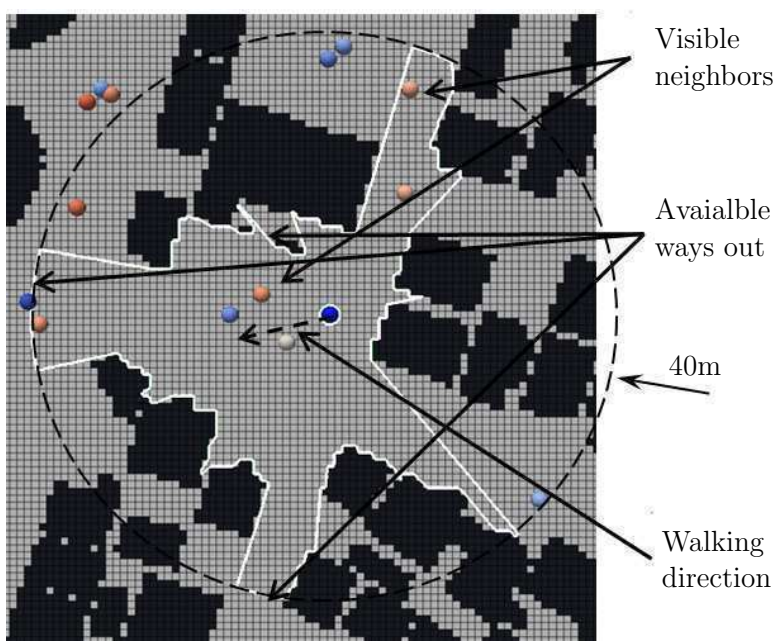

Fig.6 Agents perceive their dynamically changing environment by scanning like a radar, in high resolution.

igation for visitors agents work the same way as for residents, with the exception that they do not posses an initial mental map of the roads, which restrains them from planning their path in advance. Fig. 7 shows the initially planned path of a resident agent and the re-planned path after detecting that the previous path is blocked.

\section{(3) Path planning}

With the aim of reducing the possibility of encountering a blocked road, people may avoid taking very narrow paths, especially if the earthquake is strong enough to damage structures, walls, etc. However, the narrow roads may not be totally ignored due to several reasons. The only available path to wider roads could be narrow. Or the evacuation time can be significantly reduced if a narrow road is used for a short distance, to move from one wider road to another or even to the evacuation location. In short, people prefer to choose wider roads, depending on the availability of time or if the evacuation time (or distance traveled) can be significantly reduced. Only strict rules on the minimum or maximum path widths can be included in standard Dijkstra algorithm. It is not straight forward to do path planning with certain bias to choose wider paths, while strictly respecting time restrictions.

We modified the Dijkstra algorithm to plan paths with a given level of preference to choose wider roads and respect a given time limit. The inputs are: the starting node extracted from the agent's topological graph mental map, minimum preferred width, preferred width and level of preference. Fig. 8 shows the modified Dijkstra algorithm, with the essential changes highlighted. This modification allows agents to perform path planning with two objectives, traverse wider roads based on their preference while 


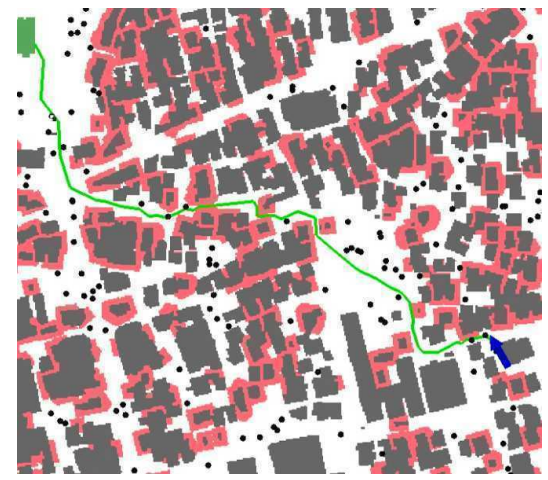

(a) Agent plans its path without knowing if the road is traversable or blocked

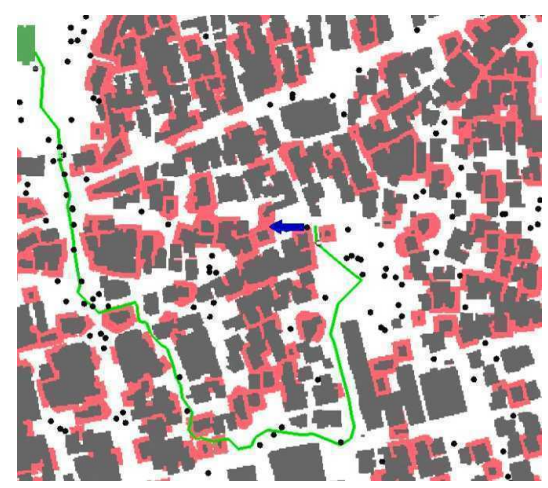

(b) Agent identifies its road as blocked and re-plans its path

Fig.7 Observed agent represented as blue arrow. (a), initial path plan as the agent doesn't know the road is blocked. (b), as the agent gets close enough and visually identifies the road as blocked, it re-plans its path.

keeping their time/distance personal constrains, i.e using this algorithm an agent with high preference of wider roads will try to use them while trying to arrive to the destination without violating his available time/distance. This is accomplished by reducing the perceived length of wider roads based on their level of preference. Roads narrower than their minimum preference are unaffected, roads wider than its preference will be perceived as reduced in length, and for the roads in between, the reduction factor will be interpolated from the current parameters, currently linear interpolation is being performed, see Fig. 8, lines 9-13. While we explore the search space of the graph, we keep track of real distance and only explore nodes further if the path distance is less than the maximum allowed distance for the agent. see Fig. 8, line 17. This maximum allowed distance is a personal goal, currently calculated using the tsunami arrival time and the agent's personal velocity, which represents the ideal case.

The effect of using different levels of preference to perform path planning with the algorithm shown in Fig. 8, are shown in Fig. 9. The preference levels the

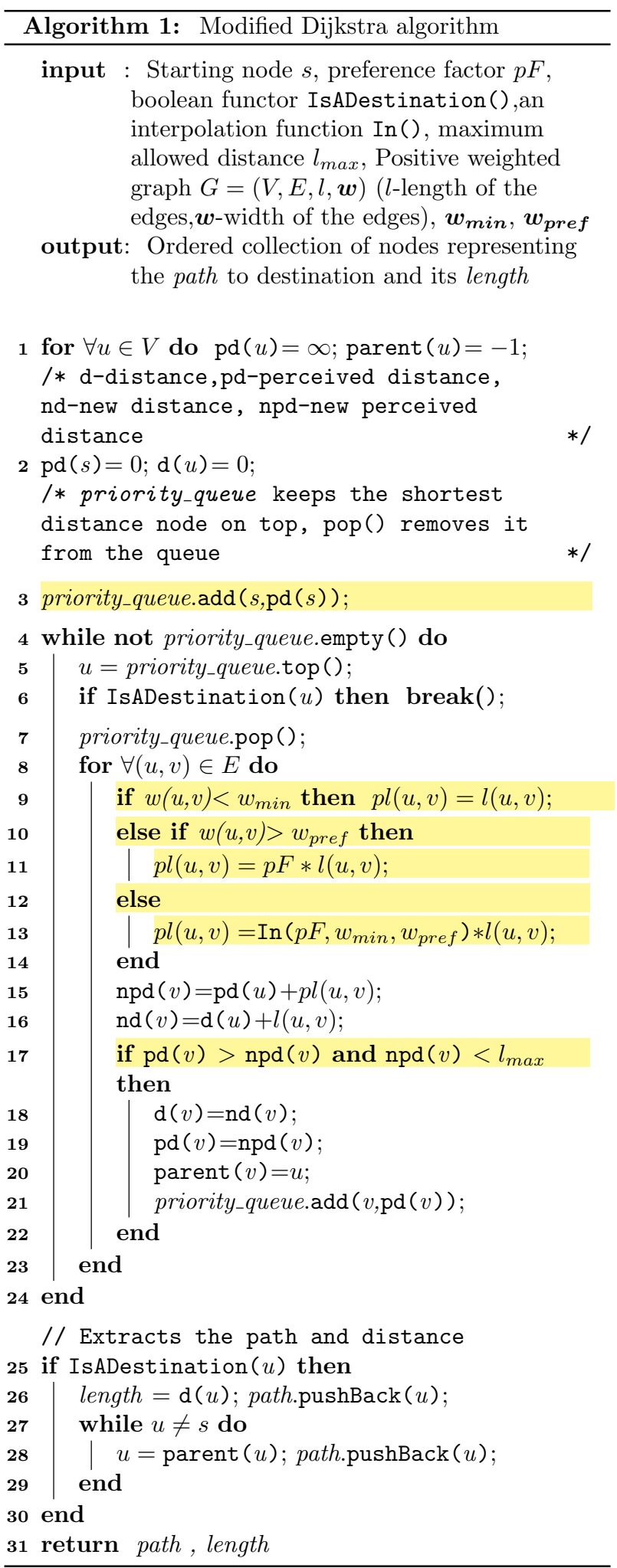

Fig.8 Preference based Dijkstra algorithm.

agents can use to plan their path range from $(0,1]$ the lower the value of the preference level, the higher in- 


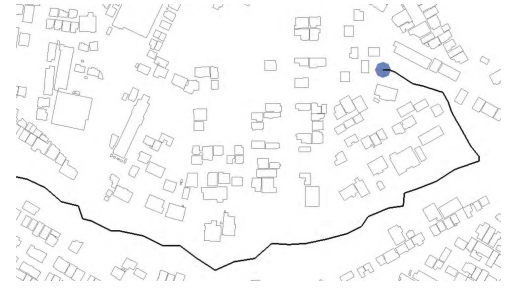

(a) preference 0.2

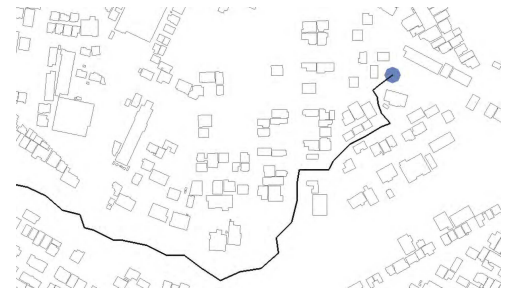

(b) preference 0.5

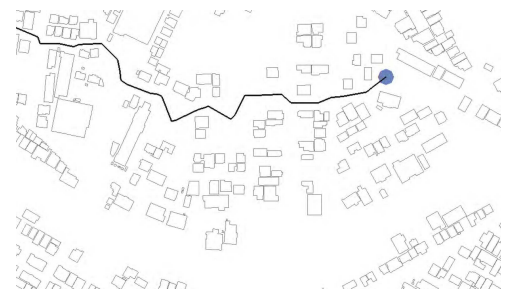

(c) preference 1.0

Fig.9 Effect of different levels of preference, evacuation area assumed to be on the left edge of the domain.

terest the agent will have to look for wider paths. A preference level of 1 will basically treat narrow and wide roads indifferently, being the paths found with this value the equivalent to the shortest path found with the unmodified Dijkstra algorithm. Although currently we have set the range of possible values to $(0,1]$ it is possible to use values above 1 to effectively avoid agents using wider paths. This could potentially be used to command official agents and rescue services to search for lost visitors in narrow neighborhoods.

\section{Demonstrative Application}

Several simulations of tsunami triggered large area evacuation, considering a high resolution model of the environment, are conducted to demonstrate the present capabilities of the model. As the domain, Kochi city environment is used due to the availability of data; GIS data of buildings and tsunami simulation data. Both the earthquake induced damages and tsunami inundation are included to demonstrate the use of the simulator in quantitative evaluation of changes in environment.

The simulations presented in this section are only for the purpose of demonstrating the capabilities of the current code, and gather readers comments and

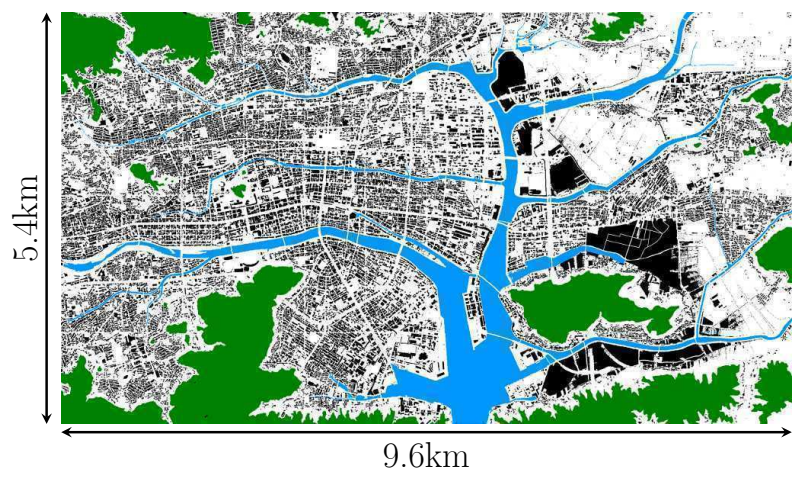

Fig.10 The simulated area. Blue, green and black color indicate water bodies, area above $30 \mathrm{~m}$ elevation and buildings, respectively.

criticisms for further improvements. None of the results presented in this section should be used for any practical purposes; the evacuation simulator has not been validated comparing observation of real evacuation, and many assumptions are made due to lack of data.

\section{(1) Common problem settings}

The $9.6 \times 5.4 \mathrm{~km}^{2}$ area shown in the Fig. 10 is considered; this area includes the most tsunami affected and possibly the most populated area of the city. A grid model of $1 \times 1 \mathrm{~m}^{2}$ resolution, which can be considered high resolution in relation to the size of the domain, is used. Due to the lack of detailed information of the paths and spaces which can be used during an emergency evacuation, all the spaces excluding buildings and water bodies are considered usable in emergency evacuations. The agents are forced not to enter narrow paths with width less than $3 \mathrm{~m}$. The agents are assumed to evacuate to areas having elevation greater than 30m, shown in green in Fig. 10.

Strong ground motion data of Kobe earthquake is used for seismic response analysis of the buildings. Buildings in the selected area is simulated with IES, using multi degree of freedom models with assumed properties. Buildings which exceeded the inter-story drift angle of 0.005 is considered damaged and the projected area of the damaged buildings are increased by $40 \%$. The inter-story drift threshold value was set as 0.005 of the inter-story height of buildings ${ }^{6), 7)}$. For the simulations which considered tsunami inundation, the grid is updated at every 5 minutes according to the inundation data provided by Takahashi et. al. ${ }^{5}$. Due to the unavailability of data, elevation of the bridges in the selected domain is assumed to be that of the ground level of the surroundings.

According to the population density of the Kochi city, the number of residents agents in the selected area is set to 57,000. These agents are generated at 
Table1 Properties of the agents

\begin{tabular}{|c|c|c|}
\hline Age & $<50$ years & $>50$ years \\
\hline Percentage & 55 & 45 \\
\hline Speed $(\mathrm{m} / \mathrm{s})$ & $1.43 \pm 0.11$ & $1.39 \pm 0.19$ \\
\hline Pre-evacuation time $(\mathrm{s})$ & \multicolumn{2}{|c|}{$1000 \pm 600$} \\
\hline Sight distance $(\mathrm{m})$ & \multicolumn{2}{|c|}{$50 \mathrm{~m}$} \\
\hline
\end{tabular}

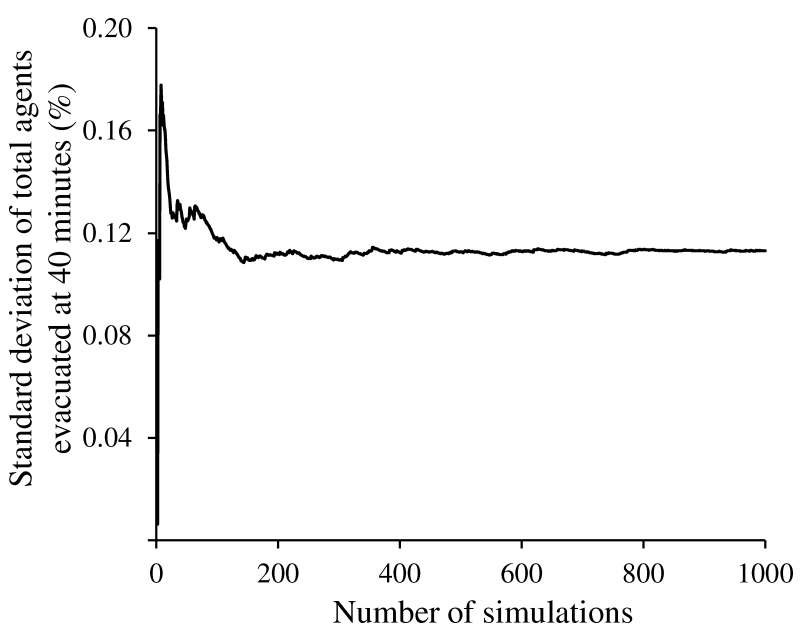

Fig.11 Standard deviation of cumulative agents evacuated at the end of 40 minutes for 1000 simulations.

random locations in the vicinity of buildings. $55 \%$ of the agents are assumed to be younger than 50 years and the rest to be older. Their properties are given in Table 1.

\section{(2) Number of cases for Monte-Carlo simulation}

A stochastic approach is necessary to evaluate the results of the simulation since the simulated phenomena involves many uncertainties modeled using random variables; e.g. properties of people and their distribution, magnitude of earthquake, tsunami inundation. Monte-Carlo simulations are conducted to account these uncertainties. In order to decide the sufficient number of cases, 1000 simulations with random distributions of agents are conducted. Fig. 11 shows the L1 norm of a vector, containing the standard deviation of the number of agents evacuated at each 30 seconds intervals, versus the number of simulations. The evacuation time is considered as the deciding factor since the end objective is reducing the total evacuation time. As is seen in Fig. 11, the standard deviation of the cumulative number of agents evacuated at 40 minutes stabilizes around 400 simulations. Hence the minimum number of required simulations is set to 400 . The results presented in this section are the average of 400 simulations.

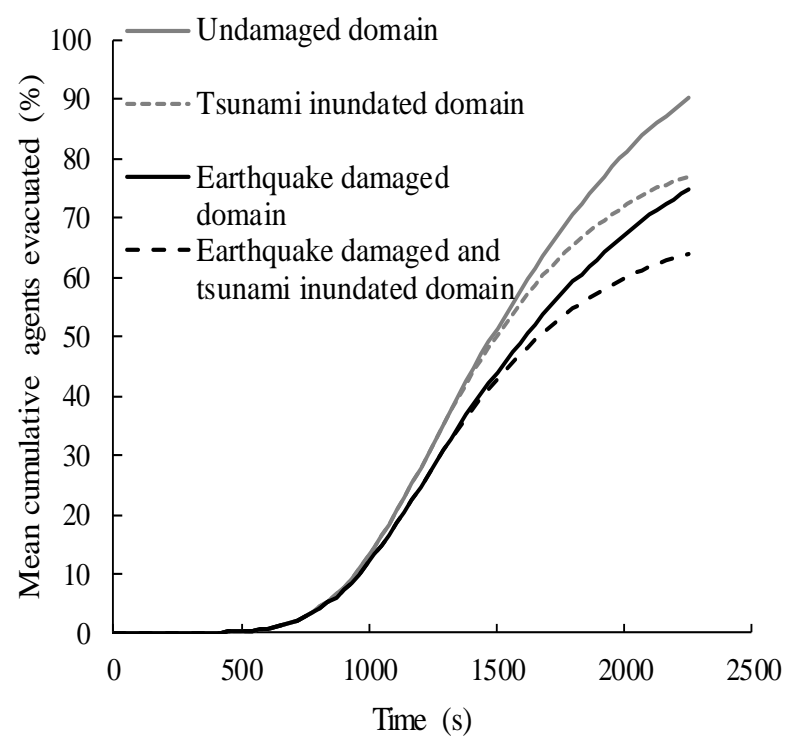

Fig.12 Comparison of evacuation in four different conditions; undamaged environment, with earthquake damages, with tsunami inundation, and with damages and inundation.

\section{(3) Effect of tsunami inundation and earthquake damages on evacuation time}

Four evacuation scenarios with different states of the environment are considered: undamaged environment; earthquake induced damages only; tsunami inundation only and with both earthquake induced damages and inundation. For all these cases, agents are generated within $20 \mathrm{~m}$ of buildings. Only one agent type (resident) was considered. 57,000 resident agents are considered.

Fig. 12 shows the cumulative number of agents evacuated, for each case. It is found that the inundation of several bridges, which are used by many agents, is the main source of reduction of number of agents evacuated. The inundation of the bridges takes place around 15 minutes or later, since the start of the simulations. Fig. 13 compares the effect of blocking the set of the bridges shown in the Fig. 14, and the effect of tsunami.

\section{a) Statistics collected to the topological graph of road network}

Agent statistics collected to the topological network is of great use in analyzing the results. While the data of each agent's location is useful in analyzing the localized effect, like crowd formation, the data collected to the graph is of great use; in comparing the effects of different scenarios, understanding how a localized effect propagates over the domain, decision making, etc. Fig. 15 shows the cumulative number of agents walked through a street segment characterized by a link of the graph in the undamaged environment. 


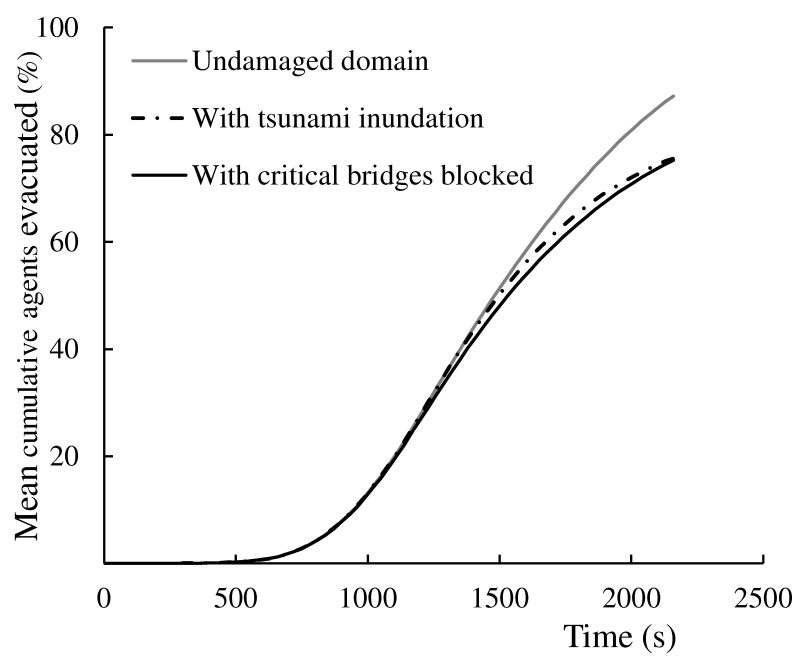

Fig.13 Comparison of the scenario with tsunami inundation and blocking the set of bridges shown in Fig 14.

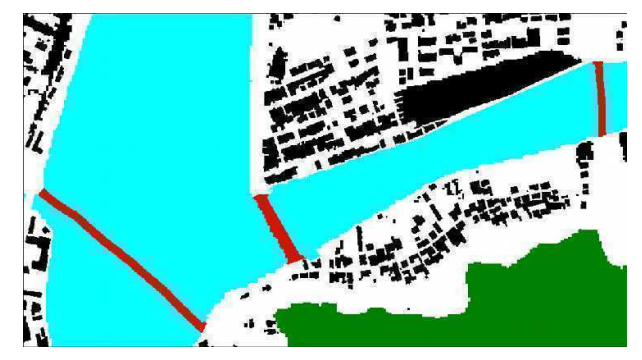

Fig.14 Blocked bridges for identified as the major cause affecting the scenario with tsunami inundation.

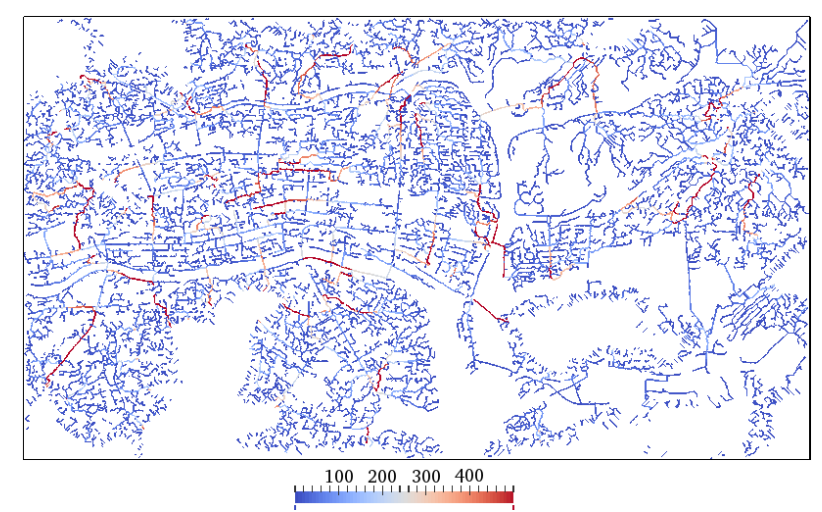

Fig.15 Cumulative number of agents that walked through a street segment characterized by link of the graph, for the scenario with undamaged environment.

\section{(4) Effect of lighting in night time evacuation}

As a possible worst case scenario, night time evacuation during a summer festival is considered. Usually, many visitors from far away places attend some of the famous festivals in coastal areas, like Yosakoi festival in Kochi city. Most of these visitors may not posses sufficient knowledge of Kochi city area. In an event

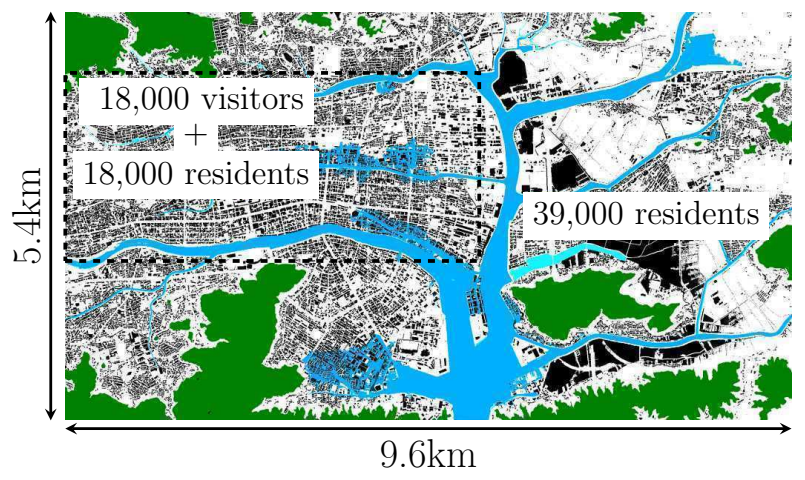

Fig.16 Domain and the distributions of agents for the evacuation during a festival.

of a night time emergency evacuation with low lighting conditions, following others is the main method of reaching a safe destination, unless some assistance is organized. It is of great importance to quantitatively identify the effect of lighting conditions on the evacuation, especially in the presence of visitors, and effective means of assisting evacuees.

\section{a) Problem setting}

It is assumed to be a full moon night, and an earthquake has damaged the environment causing a power failure. Further, 18,000 visitors and 18,000 residents are assumed to participate in a festival, spread in $14 \mathrm{~km}^{2}$ area, see Fig. 16. The remaining 39,000 residents are distributed over the whole area. The agents attending the festival are distributed across the streets and open spaces in the festival area.

The visitors are modeled to evacuate immediately, following any moving agent in its visible surrounding. The visitors have similar attributes of population ratio and agent abilities, with the distinction of no pre-evacuation time. Due to poor lighting and lack of knowledge of the surrounding, the only option for the visitors is to follow any agents evacuating. The effects of poor visibility is modeled by decreasing the speed and sight distance, according to lighting condition. Lowered sight distance affects the visitors as they evacuate by following other agents, whereas it has lesser effect on the residents since they know their surrounding.

Full moon condition is chosen due to lack of necessary information for very low lighting conditions. With full moon, 0.2lux of lighting ${ }^{12)}$ and $15 \mathrm{~m}$ sight distance $^{12), 11)}$ are assumed. To understand the effect of lighting conditions and as a possible solution to improve the evacuation process, a scenario with emergency lighting of $15 \mathrm{lux}^{13)}$ provided at spacing of $30 \mathrm{~m}$. This spacing and the level of lighting is similar to common street lighting ${ }^{13)}$. Maximum speed of agents under the considered lighting conditions are shown in Table 2. 
Table2 Effective maximum pedestrian speed under assumed lighting conditions

\begin{tabular}{|c|c|c|}
\hline \multirow{2}{*}{ Lighting conditions } & \multicolumn{2}{|c|}{ Age } \\
\cline { 2 - 3 } & $<50$ years & $>50$ years \\
\hline 15m visibility with 0.2lux & $70 \%$ & $50 \%$ \\
\hline 30m visibility with 15lux & $90.6 \%$ & $83 \%$ \\
\hline
\end{tabular}

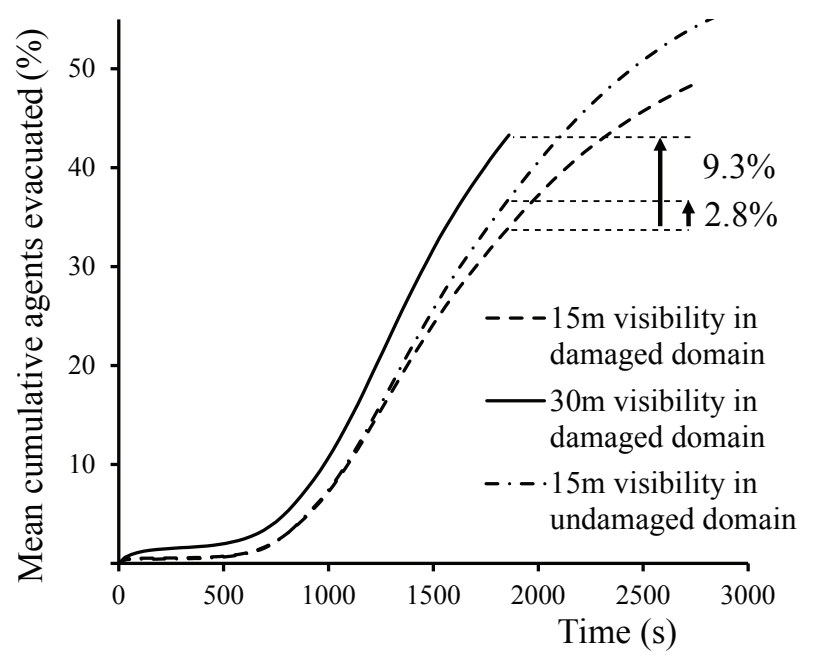

Fig.17 Cumulative number of agents evacuated; mean of 400 simulations with different agent distributions. $12,800 \mathrm{CPU}$ cores of the K-computer are used for the simulations.

It is assumed that the conditions of the environment are the same as in the case with earthquake damages and tsunami inundation, considered in the previous subsection. In the night it is assumed that people tend to take safer paths due to the uncertainty of whether the path or roads are free of obstacles due to poor visibility. In this case wider paths are considered to be safer. In order to mimic this agent behaviour the algorithm presented in Fig. 8 was used.

\section{b) Results}

The results of the two scenarios with low lighting are summarized in Fig. 17. As seen, low lighting conditions and the presence of visitors significantly lower the evacuation process, compared to the day time evacuation in ordinary day are presented in the previous subsection. Providing lighting of 15lux increases the chances of visitors to find a residents to be followed, eventually increasing the number of visitors evacuated by $5 \%$, at the beginning. To further explore the importance of lighting condition, the scenario with moon lighting is simulated excluding the effect of earthquake damages. As shown in Fig 17, improvement of lighting conditions have higher effect compared to earthquake induced damages, for this specific settings.

This simulation of the effect of lighting condition is one example which require detailed model of the environment and agents. Furthermore, the detailed agent based models are useful in getting deeper insight to the scenarios which can be simulated with a typical large area evacuation simulator.

\section{Concluding Remarks}

An agent based model for simulating large urban area evacuations, including microscopic details, is being developed. As demonstrated, the model is versatile in quantitative estimation of important scenarios which cannot be modeled with conventional models. Such a tool could be extremely useful for evacuation planners to plan evacuation measures and strategies for the long run for different areas.

Different aspects need to be considered hence the tools must be coupled with the judgment of experienced evacuation planner to reap the highest benefits.

\section{Acknowledgments}

This work was supported by JSPS KAKENHI Grant Number 24760359. Part of the results is obtained using K computer at the RIKEN Advanced Institute for Computational Science.

\section{REFERENCES}

1) Lalith, M., Melgar, L.A., Hori, M., Ichimura, T., Tanaka, S.: On the development of multi agent system for large urban area evacuation with autonomous navigation, J. of Japan Society of Civil Engineers, Ser. A2 (Applied Mechanics (AM)), Vol.69-2, I_447I_456, 2013.

2) Hori, M., Ichimura, T.: Current state of integrated earthquake simulation for earthquake hazard and disaster, J. of Seismology, pp.307-321, 2007.

3) Wijerathne, M.L.L., Melgar, A., Hori, M., Ichimura T. and Tanaka, S.: HPC enhanced large urban area evacuation simulations with vision based autonomously navigating multiagents. Procedia Computer Science, pp.1515-1524, 2013.

4) Beeson, Jong, and Kuipers: Towards autonomous topological place detection using the Extended Voronoi Graph. IEEE International Conference on Robotics and Automation (ICRA-05), pp.4373-4379, 2005.

5) Takahashi, N., Kaneda, Y., Inazawa, Y., Baba T., and Kikkojin, M.: Tsunami inundation modelling of the 2011 tohoku earthquake using threedimensional building data for Sendai, Miyagi prefecture, Japan. Tsunami Events and Lessons Learned, Advances in Natural and Technological Hazard Research, Springer, Vol.35, pp.89-98, 2014.

6) Xue, M., and Ryuzo, O.: Examination of vulnerability of various residential areas in china for earthquake disaster mititgation. In the 9th International conference on urban earthquake engineering/4th Asia con- 
ference on earthquake engineering, pp.1931-1936, Tokyo, 2012.

7) Theodore, V., Galambos, Ellingwood, B.: Serviceability Limit States: Deflection, Journal of Structural Engineering, ASCE, pp.67-84, 1986.

8) URL http://www.pref.kochi.lg.jp/soshiki/111601/ jinkomondai.html.

9) Richard W. Bohannon: Comfortable walking speed of adults aged 20-79 years: reference values and determinants. Age and Ageing, Vol.26, pp.15-19, 1997.

10) Dhulam, R., Lalith, M., Hori, M., Ichimura, T. and Tanka, S.: A study on effectiveness of using officials for reducing pre-evacuation time in a large area based on multi agent simulations. In 9th International conference on urban earthquake engineering / 4th Asia conference on earthquake engineering. pp.16581665, Tokyo, 2012.
11) Nichols, T. F. and Powers, T. R.: Moonlight and night visibility. U.S. Army Training Center Human Research Unit, Presidio of Monterey, California, January 1964. 11) Use-ip ltd. Lux light level chart, Pdf.

12) Honeywell. Emergency lighting design guide, Pdf, February 2014

13) Arizona transportation department, City of Glendale. Street lighting manual. Pdf, 2006

14) Ouellette, M. J., Rea, M. S.: Illuminance Requirements for Emergency Lighting, Journal of the Illuminating Engineering Society, pp.37-42, 1989.

(Received June 20, 2014) 